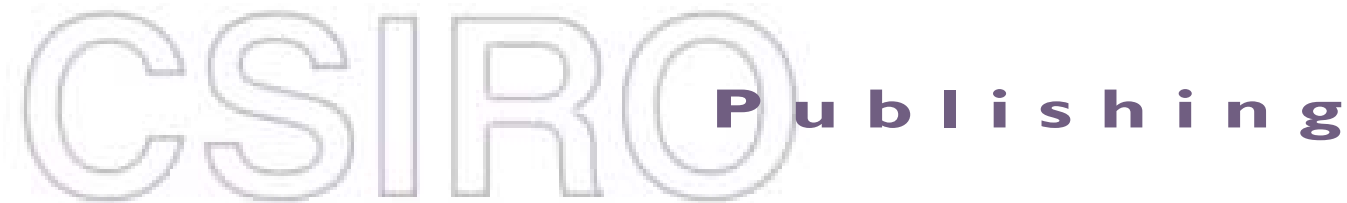

\section{Publications of the Astronomical Society of Australia}

Volume 19, 2002

(C) Astronomical Society of Australia 2002

An international journal of astronomy and astrophysics



For general enquiries and subscriptions, please contact: CSIRO Publishing PO Box 1139 (150 Oxford St)

Collingwood, Vic. 3066, Australia

Telephone: +6139662 7666

Fax: +61 396627555

Email: publishing.pasa@csiro.au

C S I RO

PUBLISHING Published by CSIRO Publishing

for the Astronomical Society of Australia

www.publish.csiro.au/journals/pasa 


\title{
Interstellar Scintillation Explains the Intraday Variations in the Linear Polarisation of Quasar PKS 0405-385 at cm-wavelengths
}

\author{
Barney Rickett ${ }^{1}$, Lucyna Kedziora-Chudczer ${ }^{2}$ and David L. Jauncey ${ }^{3}$ \\ ${ }^{1}$ University of California San Diego, La Jolla, CA92093, USA \\ bjrickett@ucsd.edu \\ ${ }^{2}$ Australia Telescope National Facility and Anglo-Australian Observatory, Sydney, Australia \\ lkedzior@atnf.csiro.au \\ ${ }^{3}$ Australia Telescope National Facility, Sydney, Australia \\ djauncey@atnf.csiro.au \\ Received 2001 September 3, accepted 2002 February 13
}

\begin{abstract}
During its burst of intraday variation (IDV) in June 1996 quasar PKS 0405-385 exhibited rapid changes in the Stokes parameters $Q$ and $U$ that were even faster than the $1 \mathrm{hr}$ timescale changes in $I$. Autoand cross-correlation analyses of $I, Q$, and $U$ at $8.6 \mathrm{GHz}$ show systematic relations between the variations in $I, Q$, and $U$. These are well modelled by interstellar scintillation (ISS) of a $14 \times 20 \mu$ as source, with about 180 degree rotation of the polarisation angle along its long dimension. This success in explaining the remarkable IDV in polarisation confirms that ISS gives rise to the IDV in this quasar.
\end{abstract}

Keywords: galaxies: nuclei — galaxies: jets — radio continuum: galaxies — scattering — galaxies: ISM

\section{Polarised IDV}

Many IDV sources show significant levels of varying linear polarisation (e.g. Quirrenbach et al. 1989; Macquart et al. 2000), often changing more rapidly than the total flux. Further, Gabuzda et al. (2000a,b) have found changes in polarised sub-structure in three AGN on timescales as short as $12 \mathrm{hr}$. While intrinsic models have been proposed for such variations, Rickett et al. (1995, hereafter R95) and Qian et al. (2001) have argued that both intensity and polarisation changes could be explained as ISS. The goal here is to present an ISS interpretation of the rapid variations in linearly polarised flux density for the quasar PKS 0405-385, during its episode of very rapid IDV (Kedziora-Chudczer et al. 1997, hereafter KC97). Figure 1 shows a $12 \mathrm{hr}$ time series of $I, Q$, and $U$ on June 8, 1996 at $8.6 \mathrm{GHz}$ from the Australia Telescope Compact Array.

\section{ISS in Stokes Parameters}

In describing the relation between variations in intensity and linear polarisation, we distinguish between changes in the degree $(p)$ and/or angle $(\chi)$ of the linear polarisation and the simpler case of changes in the flux density of a polarised source, for which $p$ and $\chi$ remain constant. The data in Figure 1 represent rapid and substantial variations in $p$ and $\chi$. However, instead of plotting $p, \chi$ we plot $Q, U$, since they add linearly for independent fluctuating or steady components. As a special case the polarised signal is considered as the sum of a steady component and a single component of varying flux, but fixed $p$ and $\chi$. The $Q$ variations should then be modelled as $Q(t)=Q_{o}+m_{q} \delta I(t)$, where $Q_{o}$ is the total average $Q, \delta I(t)$ is the deviation of $I$ from its mean, and $m_{q}=p \cos (2 \chi)$, with a similar relation for $U(t)(\mathrm{R} 95)$.
Such relations should apply for ISS of an intrinsically steady isolated point source, since the diffraction patterns in $Q, U(\& V)$ should be identical to that in $I$ with added constants. This conclusion comes from considering the phase difference between the right and left circular polarisation modes of propagation in the ISM, which is governed by the rotation measure. For PKS 0405-385 we observe $R M \sim 70 \mathrm{rad} \mathrm{m}^{2}$, which at $8.6 \mathrm{GHz}$ gives $<0.2 \mathrm{rad}$ phase difference between left and right. However, for scintillation in $p$ or $\chi$, what matters is the change in this phase difference across the Fresnel scale $\left(r_{f}=\sqrt{\lambda z / 2 \pi}\right.$ for scattering at distance $z$ from the observer), which turns out to be only about one millionth of a radian. This is negligible compared to the $0.1-0.5 \mathrm{rad}$ difference in total propagation phase across the Fresnel scale, typical of the PKS 0405-385 observations which are in weak ISS (KC97). The smooth curves overplotted with observed $Q, U$ in Figure 1 come from the linear relations, mentioned above, with the best fits for the constants $\left(m_{q}, m_{u}, Q_{o}, U_{o}\right)$; however, the curves clearly fail to fit the observations and a more complex model is needed.

Consequently, we ask whether the addition of several components with quasi-independent flux density variations, but each maintaining its own fixed $p, \chi$, could produce the rapid changes seen in $Q, U$. This could apply for intrinsic variations across the source in which the magnetic structure remained constant, and it is also expected for ISS of a source which is extended, since the ISS variations from sub-components are only partially correlated with each other. Thus if there is structure in $p$ or $\chi$ across the source we must consider the summation of mutiple terms each of fixed $p$ and $\chi$, but for which the net $p$ and $\chi$ may vary rapidly.

In order to characterise the relations observed between $I(t), Q(t)$, and $U(t)$, we computed their auto- and 

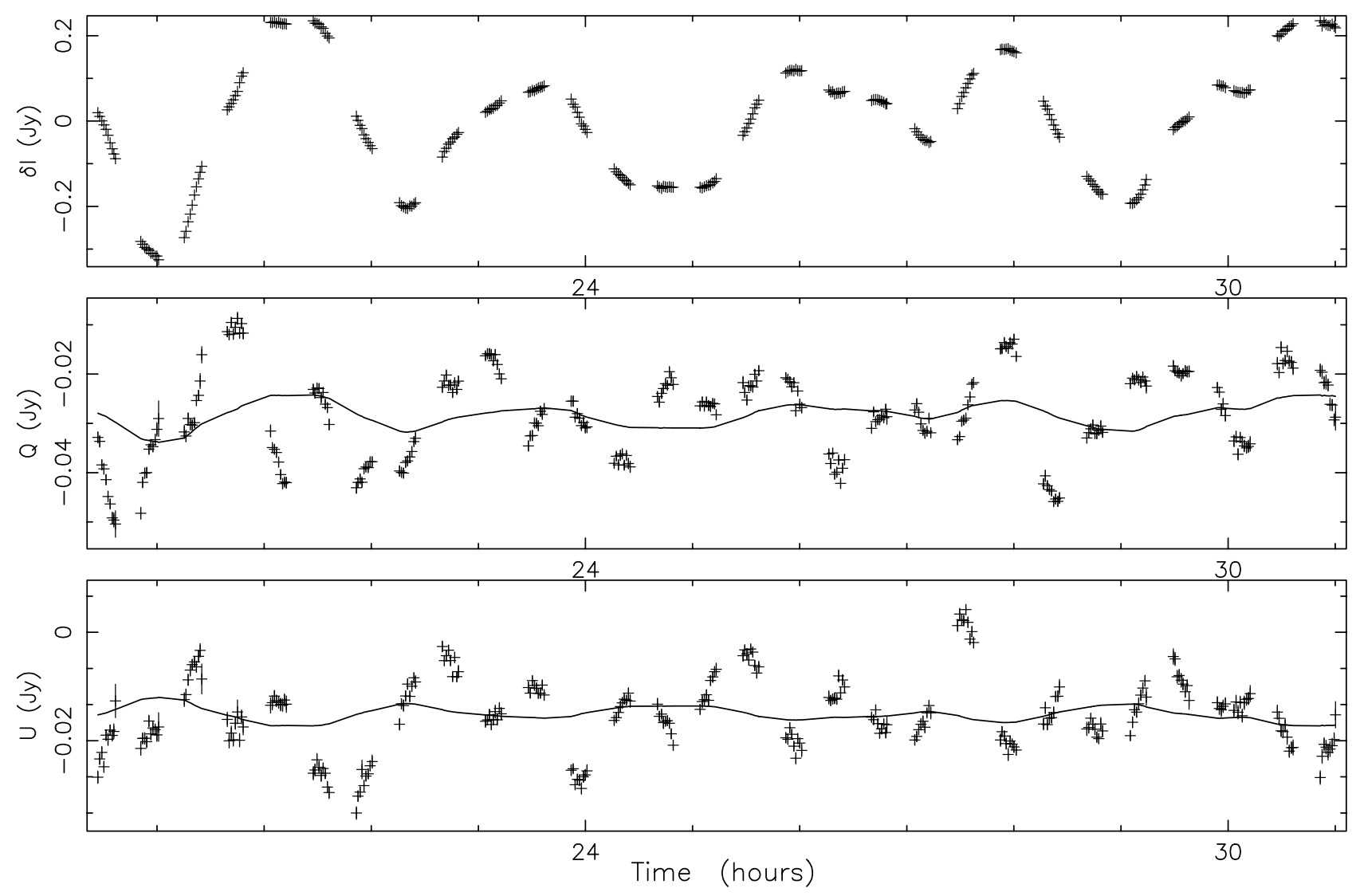

Figure 1 Time series of $\delta I, Q$ and $U$ on June 8, 1996 at 8.6 GHz from PKS 0405-385 vs time in hours relative to 0 hr UT on June 7. The error bars are mostly smaller than the plotting symbols. The lines are linear fits of the variations in $Q, U$ to those in $I$, as described in the text; evidently there are more rapid changes in $Q, U$ than in $I$.

cross-correlations. Although a low but significant level of circular polarisation was also observed at $8.6 \mathrm{GHz}$, we concentrate here on interpreting the linear polarisation, for which the signal to noise ratio is very high. In the following we summarise the theory of the $I, Q, U$ correlations, which were also presented by Rickett (2001) and a closely related analysis by Medvedev (2000). The theory applies for weak or strong ISS and assumes a simple screen geometry; results for an extended scattering medium will be given in a more complete paper, in preparation.

Consider a scattering screen of thickness $\delta z$ at distance $z$ from an observer who receives polarised waves from a source with brightness distribution $B_{I}(\vec{\theta})$ in $I$. Let $\Delta I_{o}(\vec{s})$ be the pattern of intensity fluctuation about its mean at transverse location $\vec{s}$ for a unit flux density point source located on the $\mathrm{z}$ axis. The net intensity is the sum of shifted terms weighted by each brightness element:

$$
\Delta I(\vec{s})=\iint \Delta I_{o}(\vec{s}-z \vec{\theta}) B_{I}(\vec{\theta}) d^{2} \vec{\theta},
$$

where the angles are all small and infinite limits are implied in this and subsequent integrals. Exactly similar equations can be written for $\Delta Q$ and $\Delta U$ for a source with polarised brightness functions $B_{Q}(\vec{\theta})$ and $B_{U}(\vec{\theta})$, since the ISS in, say, $Q$ due to each source element is given simply by $\Delta I_{o}(\vec{s}-z \vec{\theta}) B_{Q}(\vec{\theta}) d^{2} \vec{\theta}$.
For a point source, ISS caused by a screen is statistically homogeneous and has a spatial autocorrelation which depends only on the spatial separation $\vec{\sigma}$, not on absolute position $\vec{s}$ :

$$
C_{o o}(\vec{\sigma})=\left\langle\Delta I_{o}(\vec{s}) \Delta I_{o}(\vec{s}+\vec{\sigma})\right\rangle
$$

Cross-correlation versus spatial offset $\vec{\sigma}$ can be defined for each pair of observed Stokes' parameters; e.g. for I and $Q$ :

$$
C_{I Q}(\vec{\sigma})=\langle\Delta I(\vec{s}) \Delta Q(\vec{s}+\vec{\sigma})\rangle
$$

Substituting equation (1) and the parallel equation for $\Delta Q$ one obtains

$$
C_{I Q}(\vec{\sigma})=\iint C_{o o}(\vec{\sigma}-z \vec{\alpha}) R_{I Q}(\vec{\alpha}) d^{2} \vec{\alpha},
$$

where

$$
R_{I Q}(\vec{\alpha})=\iint B_{I}(\vec{\beta}-\vec{\alpha} / 2) B_{Q}(\vec{\beta}+\vec{\alpha} / 2) d^{2} \vec{\beta}
$$

A version of equations (4) and (5) with subscripts $I I$ in place of $I Q$ gives the standard result for $C_{I I}$ in terms of $R_{I I}$ (Little \& Hewish 1966). Evidently there are similar results for the six auto- and cross-correlations $(I I, Q Q, U U, I Q, I U, Q U)$. In these equations the 
observable correlations are given as the convolution of a brightness correlation function, such as $R_{I Q}$, with a 'resolution function' $C_{o o}$, which is the autocorrelation of $I$ due to an isolated point source. Observationally, we are

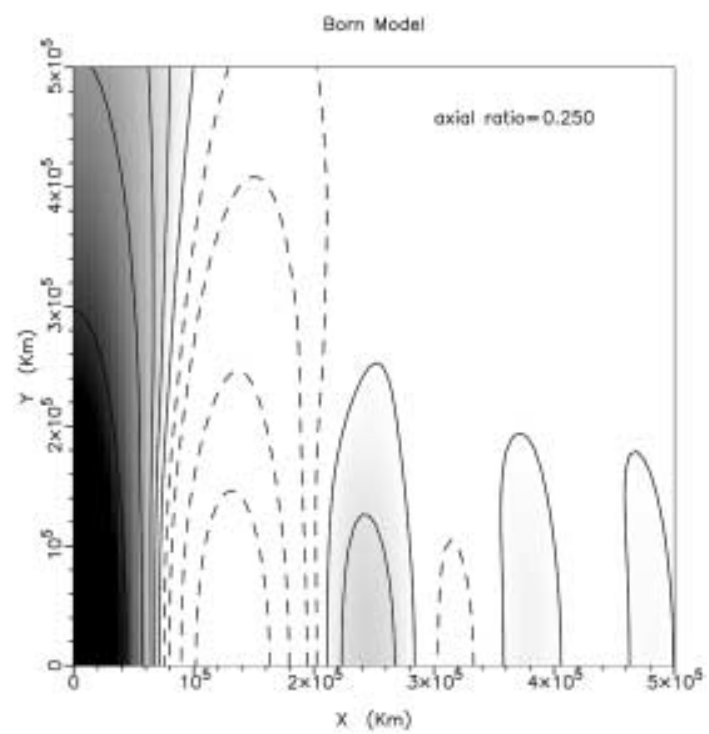

Figure 2 Contour plot of $C_{o o}(\vec{\sigma})$ in a single quadrant (function is symmetric about both axes). The dashed contours are negative at $(-0.05,-0.1,-0.2,-0.3)$ and show the deep negative lobes; the shaded solid contours visible are at $(0.4,0.2,0.1,0.05,0.0)$. Here $r_{f}=6.7 \times 10^{4} \mathrm{~km}$.
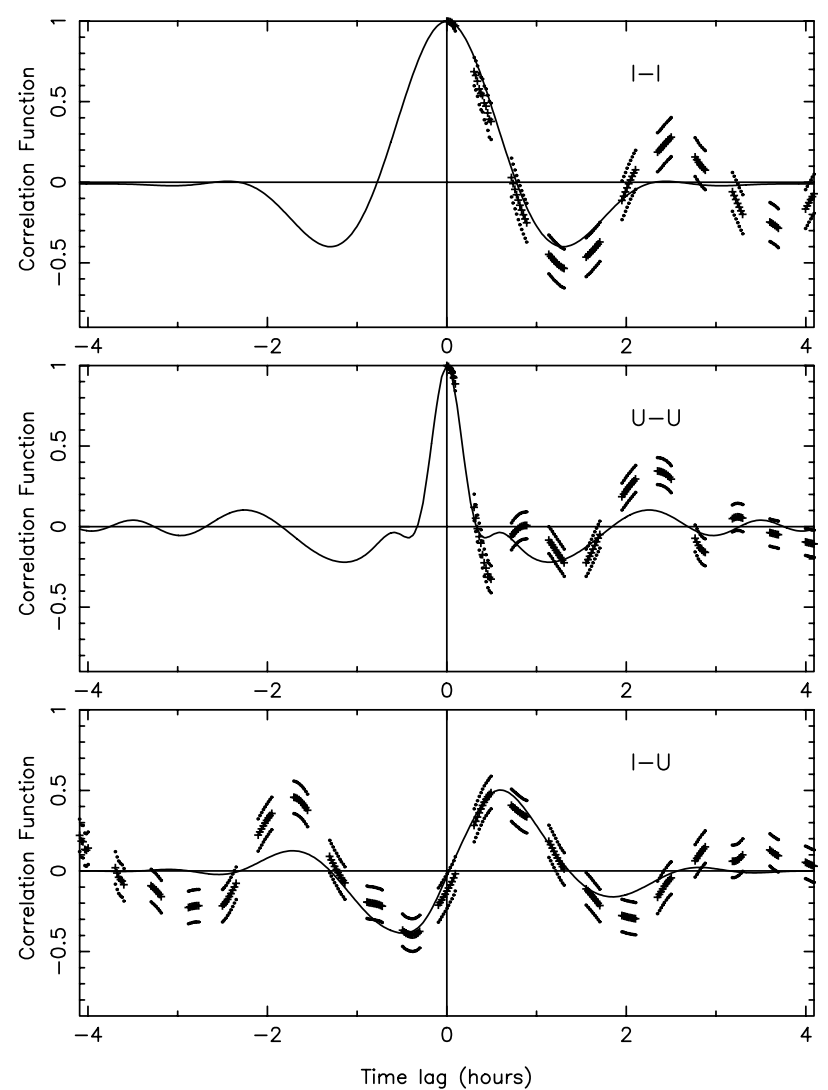

able to probe the six brightness correlation functions with a common resolution function.

These relations apply for all branches of ISS (weak or strong), but at present no extragalactic radio source has been found with a small enough angular size to exhibit diffractive ISS. Consequently, we are concerned with refractive ISS in strong scintillation or with weak ISS. In both cases expressions for $C_{o o}$ are most simply given in the wavenumber domain as a cross-spectrum (such as in Appendix B of R95). The cross-spectrum for ISS in $I$ and $Q$, which is the Fourier transform of $C_{I Q}(\vec{\sigma})$, is given by equations analogous to those in Rickett (2002) for anisotropic scattering.

In isotropic weak scintillation the form of $C_{o o}(\vec{\sigma})$ is a smooth circular function of width $r_{f}$ peaking at the origin. When the fine structure of the plasma is anisotropic, the 'resolution function' also becomes anisotropic, as shown in Figure 2. As described by Rickett (2002; Figure 3), anisotropy in the plasma is the only way to match the observed shape of the autocorrelation of $I$ for PKS 0405385. In the direction perpendicular to the filamentary plasma structures $C_{o o}(\vec{\sigma})$ has a width of about $r_{f} / \sqrt{(2)}$ and also has pronounced negative 'lobes' along this short axis, which are absent along the other axis. This is illustrated in the top left panel of Figure 3, in which an axial ratio $0.25: 1$ is assumed with the velocity of the pattern approximately perpendicular to the plasma filaments.
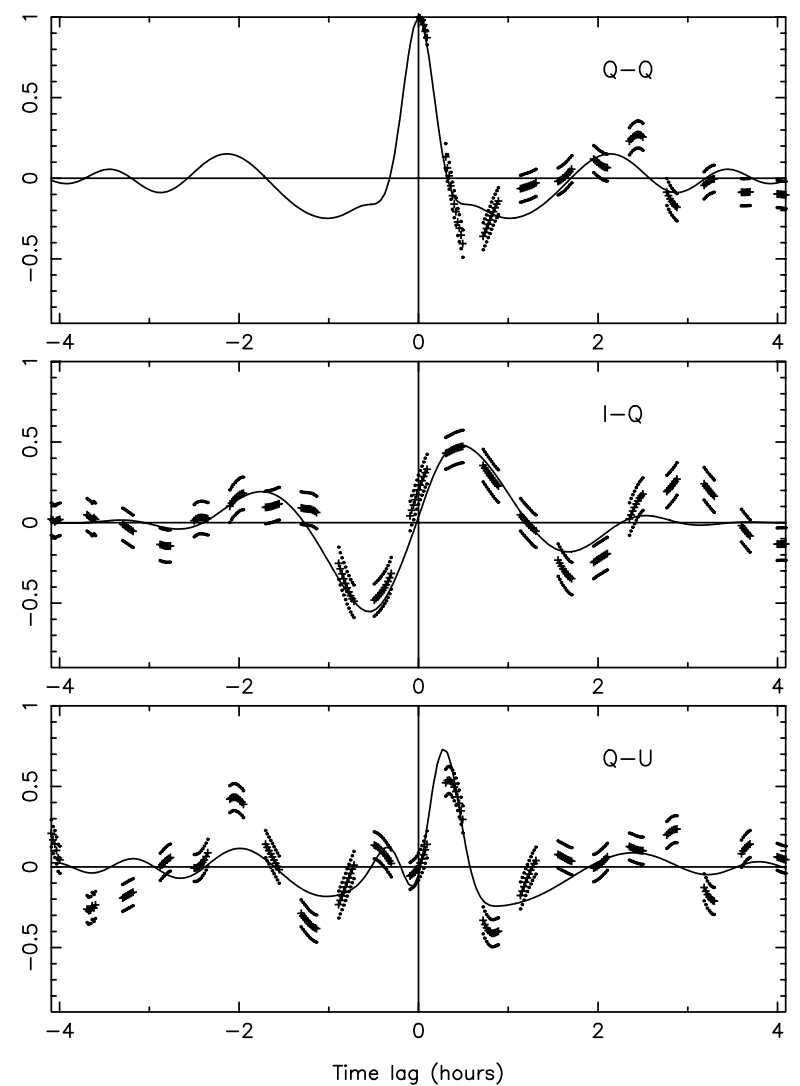

Figure 3 Points are auto- and cross-correlations of $I, Q, U$ with $\pm 1 \sigma$ errors plotted as dots above and below. The data are the average from June 8-10, 1996 from PKS $0405-385$ at $8.6 \mathrm{GHz}$. The solid line is the weak ISS model, after fitting a specific source model, as described in the text. The fit was done with a triangular weight going to zero at $\pm 3 \mathrm{hr}$. 


\section{Results and Discussion}

Figure 3 compares the correlations in $I, Q, \& U$ at $8.6 \mathrm{GHz}$ with weak ISS theory. The theoretical equations, in terms of spatial offsets, are used to model the six temporal autoand cross-correlations (at time lag $\tau$ ) by setting $\vec{\sigma}=\boldsymbol{V}_{\text {iss }} \tau$, for the motion of the observer through a time stationary scintillation pattern at velocity $\boldsymbol{V}_{\text {iss. }}$. In general we do not know the orientation of this velocity vector, but, as mentioned above, the observed shape of the autocorrelation of $I$ constrains $V_{\text {iss }}$ to be nearly at right angles to the filamentary structure.

Rickett (2002) also argues that the ISS of PKS 0405-385 can be modelled by a thin layer at $25 \mathrm{pc}$ from the Earth with a Kolmogorov density spectrum and axial ratio of 0.25 . Here we kept these parameters fixed for such a screen model and examined source models consisting of sums of circular Gaussian components each with a fixed value of $p$ and $\chi$. We performed a fitting process to find a source model that fits the observed correlation functions. With only two source components the best result provided a good fit to all functions except the cross correlation $C_{Q U}$. However, with three components all six correlations can be matched as shown by the particular example of Figure 3. Here the (reduced) $\chi$-squared sum is 1.7 , where a value near 1.0 would be expected for a statistically good fit. Note that the maxima and minima in the auto- and cross-correlations do not die away with increasing time lag, and that substantial differences remain between theory and observation. The computed $\chi$-squared depends directly on the errors in the correlation functions, which are hard to estimate. In fact the systematic uncertainties in the normalisation of the $\chi$-squared are sufficiently large that, at this stage of the analysis, we accept 1.7 as a satisfactory fit, and have not attempted a more complex model. It should be noted that the source model is

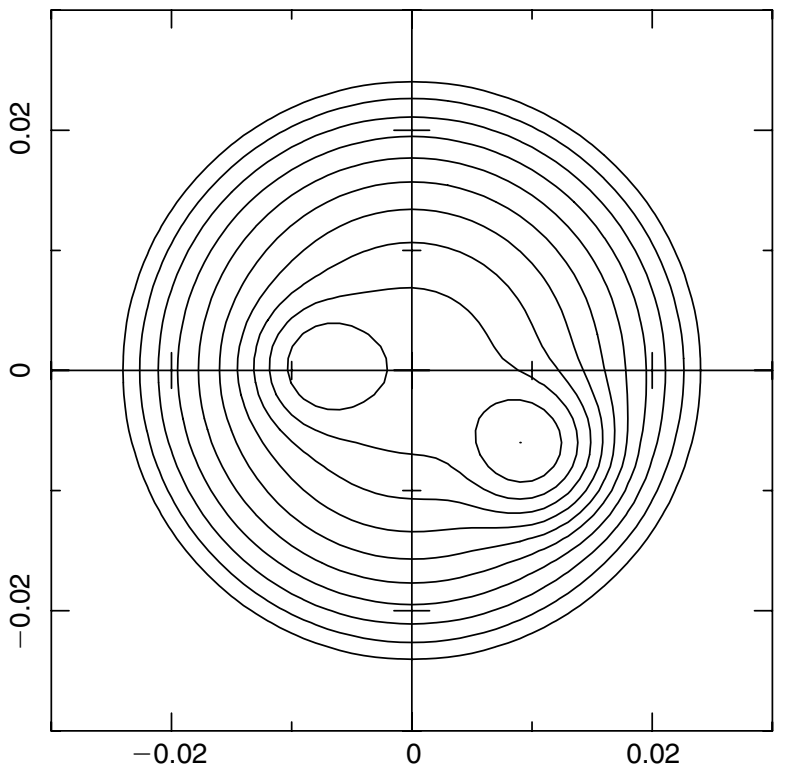

by no means unique and that a large (and complex) region in the multi-dimensional parameter space provides similar fits. In order to constrain it further we must improve the modelling of the observed rms in $I$ and $P=\sqrt{Q^{2}+U^{2}}$, which in the analysis here (arbitrarily) assumes $60 \%$ of the total flux density is variable. The other $40 \%$ must be in a component considerably more extended, say by 5 times, making its diameter 0.1 mas or larger, which would remain unresolved even with VSOP.

Figure 4 shows the source structure in $I$ and $P$ for the model of Figure 3 with the polarisation angle shown by a line of fixed length. The highest contour in $I$ corresponds to a brightness temperature near $4 \times 10^{13} \mathrm{~K}$, with levels decreasing by factors of $63 \%$. However, the brightness temperature scale is uncertain, because it depends on the unknown percentage of the flux density that is variable. We are working to constrain this by a more complete analysis, including an explicit fit to the observed rms flux density (Rickett, Kedziora-Chudczer, \& Jauncey 2002). We further note that the auto- and cross-correlations of $I, Q, U$ at $4.8 \mathrm{GHz}$ do not show the systematic behaviour seen at $8.6 \mathrm{GHz}$ and have $20 \%$ lower rms in $Q$ and $U$, and yet $30 \%$ higher rms in $I$. We suggest that the explanation may be that there is more internal depolarisation in the source at the lower frequencies, reducing the amplitude of IDV in polarised flux and changes in the $I, Q, U$ relations.

We conclude here that a model for the polarised IDV has been found assuming weak ISS from anisotropic plasma structures in a thin layer about $25 \mathrm{pc}$ from the Earth with a plausible source structure. Thus the IDV in $p$ and $\chi$ can indeed be explained as the superposition of ISS from three components creating a $14 \times 20 \mu$ as source with $\sim 180^{\circ}$ rotation in $\chi$ along its long dimension. This result also highlights the need to understand the propagation conditions in the local ionised interstellar medium.

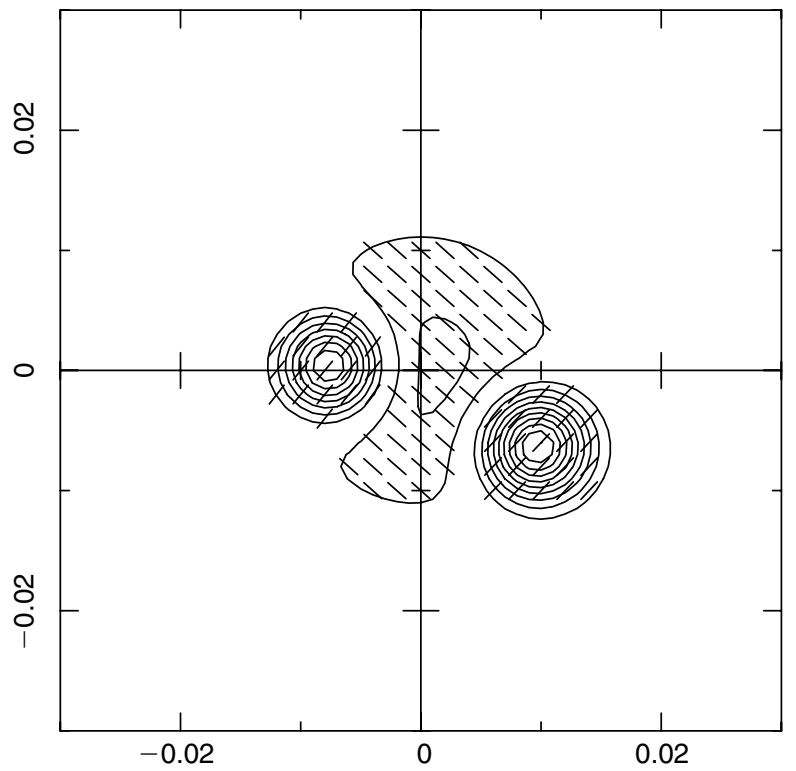

Figure 4 Contours of brightness in $I$ (left) and $P$ (right) for the source model used in Figure 3; the angular unit scale is in milliarcsec with the x-axis parallel to $\boldsymbol{V}_{\text {iss. }}$. The bars show the polarisation angle where $P$ is greater than $10 \%$ of its maximum. 
As concluded by Rickett (2002) the need to invoke a scattering region as close as $25 \mathrm{pc}$ from the Earth reduces the required peak brightness temperature to $\lesssim 4 \times 10^{13} \mathrm{~K}$, which implies a Doppler factor $\lesssim 100$. Though this is less than 1000 implied by ISS at the expected distance of $500 \mathrm{pc}$, it remains well above the values provided by VSOP (Hirabayashi et al. 2000), which could however be lower limits if there are unresolved components. VSOP observations of PKS 0405-385 were made but at a time after the IDV had died away; however, VLBI observations in June 1996 (Kedziora-Chudczer et al. 2001) indicated over $85 \%$ of the flux was unresolved at a resolution of about $1 \mathrm{mas}$ at $8.6 \mathrm{GHz}$, which is consistent with the model from our ISS interpretation. We conclude that PKS 0405-385 is a relativistic jet at the high end of the range allowed by VLBI observations. We can, crudely, estimate the angular separation of an emerging jet component from the core over the two months during which the IDV was observed. This depends on the angle of the jet to the line of sight, which we take to be near the peak of the relativistic beam; with a Doppler factor in the range 50-100 this gives an angular separation of 0.1-0.2 mas over two months. This is large enough to quench the scintillations and so could explain the disappearance of the IDV.

\section{Acknowledgments}

The Australia Telescope Compact Array is part of the Australia Telescope, which is funded for operation as a
National Facility by CSIRO. BJR thanks the US NSF for support under grant AST 9988398 and thanks Ron Ekers, the Director, for support while visiting the ATNF.

\section{References}

Gabuzda, D. C., Kochenov, P., Cawthorne, T. V., \& Kollgard, R. 2000a, MNRAS, 313, 627

Gabuzda, D. C., Kochenov, P., Kollgard, R., \& Cawthorne, T. V. 2000b, MNRAS, 315, 229

Hirabayashi, H., et al. 2000, PASJ, 52, 997

Kedziora-Chudczer, L., Jauncey, D. L., Wieringa, M. H., Walker, M. A., Nicholson, G. D., Reynolds, J. E., \& Tzioumis, A. K. 1997, ApJ, 490, L9 (KC97)

Kedziora-Chudczer, L., et al. 2001, in Particles and Fields in Radio Galaxies, ASP Conference Series 250, eds R. A. Laing, \& K. M. Blundell (San Francisco: ASP)

Little, L. T., \& Hewish, A. 1966, MNRAS, 134, 221

Macquart, J.-P., Kedziora-Chudczer, L., Rayner, D. P., \& Jauncey, D. L. 2000, ApJ, 538, 623

Medvedev, M. 2000, ApJ, 532, L63

Qian, S. J., Witzel, A., Kraus, A., Krichbaum, T. P., \& Zensus, A. 2001, A\&A, 367, 770

Quirrenbach, A., Witzel, A., Krichbaum, T., Hummel, C. A., Alberdi, A., \& Schalinski, C. J. 1989, Nature, 337, 442

Rickett, B. J. 2001, Ap\&SS, 278, 129

Rickett, B. J. 2002, PASA, 19, 100

Rickett, B. J., Kedziora-Chudczer, L., \& Jauncey, D. L. 2002, ApJ, submitted

Rickett, B. J., Quirrenbach, A., Wegner, R., Krichbaum, T., \& Witzel, A. 1995, A\&A, 293, 479 (R95) 